OPEN ACCESS

Edited by:

Sven Haller,

Rive Droite SA, Switzerland

Reviewed by:

Ravinder Nagpal,

Wake Forest School of Medicine,

United States

Drozdstoy Stoyanov Stoyanov, Plovdiv Medical University, Bulgaria

${ }^{*}$ Correspondence:

Federica Pinna

fedepinna@inwind.it

tThese authors have contributed equally to this work

Specialty section:

This article was submitted to Neuroimaging and Stimulation,

a section of the journal

Frontiers in Psychiatry

Received: 21 July 2020 Accepted: 15 December 2020 Published: 12 January 2021

Citation:

Sani G, Manchia M, Simonetti A, Janiri D, Paribello P, Pinna F and Carpiniello $B$ (2021) The Role of Gut Microbiota in the High-Risk Construct of Severe Mental Disorders: A Mini Review. Front. Psychiatry 11:585769.

doi: 10.3389/fpsyt.2020.585769

\section{The Role of Gut Microbiota in the High-Risk Construct of Severe Mental Disorders: A Mini Review}

\author{
Gabriele Sani ${ }^{1,2 \dagger}$, Mirko Manchia ${ }^{3,4,5+}$, Alessio Simonetti ${ }^{6,7}$, Delfina Janiri ${ }^{1,2,8}$, \\ Pasquale Paribello ${ }^{3,4}$, Federica Pinna ${ }^{3,4 *}$ and Bernardo Carpiniello ${ }^{3,4}$
}

${ }^{1}$ Fondazione Policlinico Universitario "Agostino Gemelli" Istituto di ricovero e cura a carattere scientifico (IRCCS), Rome, Italy, ${ }^{2}$ Section of Psychiatry, Department of Neuroscience, Università Cattolica del Sacro Cuore, Rome, Italy, ${ }^{3}$ Section of Psychiatry, Department of Medical Sciences and Public Health, University of Cagliari, Cagliari, Italy, ${ }^{4}$ Unit of Clinical Psychiatry, University Hospital Agency of Cagliari, Cagliari, Italy, ${ }^{5}$ Department of Pharmacology, Dalhousie University, Halifax, NS, Canada, ${ }^{6}$ Menninger Department of Psychiatry and Behavioral Sciences, Baylor College of Medicine, Houston, TX, United States, ${ }^{7}$ Department of Neurology and Psychiatry, Sapienza University of Rome, Rome, Italy, ${ }^{8}$ Department of Psychiatry, Icahn School of Medicine at Mount Sinai, New York, NY, United States

Severe mental disorders (SMD) are highly prevalent psychiatric conditions exerting an enormous toll on society. Therefore, prevention of SMD has received enormous attention in the last two decades. Preventative approaches are based on the knowledge and detailed characterization of the developmental stages of SMD and on risk prediction. One relevant biological component, so far neglected in high risk research, is microbiota. The human microbiota consists in the ensemble of microbes, including viruses, bacteria, and eukaryotes, that inhabit several ecological niches of the organism. Due to its demonstrated role in modulating illness and health, as well in influencing behavior, much interest has focused on the characterization of the microbiota inhabiting the gut. Several studies in animal models have shown the early modifications in the gut microbiota might impact on neurodevelopment and the onset of deficits in social behavior corresponding to distinct neurosignaling alterations. However, despite this evidence, only one study investigated the effect of altered microbiome and risk of developing mental disorders in humans, showing that individuals at risk for SMD had significantly different global microbiome composition than healthy controls. We then offer a developmental perspective and provided mechanistic insights on how changes in the microbiota could influence the risk of SMD. We suggest that the analysis of microbiota should be included in the comprehensive assessment generally performed in populations at high risk for $\mathrm{SMD}$ as it can inform predictive models and ultimately preventative strategies.

Keywords: microbiome, schizophrenia, depression, genomics, animal models, autism spectrum disorder, Shannon index, alpha diversity

\section{INTRODUCTION}

Severe mental disorders (SMD), including schizophrenia, bipolar disorder and major depressive disorder, are commonly occurring psychiatric conditions exerting an enormous toll on society (1). The 2010 estimate of Gustavsson and co-authors showed that cumulatively direct and indirect costs associated to SMD amount at $\sim € 140$ billion per year in Europe (2). Several factors, other 
than the elevated prevalence in the general population, determine the substantial burden of SMD. First, their longitudinal trajectory start during late adolescence-young adulthood with a lifelong duration in the vast majority of cases $(3,4)$. Second, the clinical course of SMD is often chronic with recurrent episodes of psychopathological disturbances and presence of persistent residual symptoms that significantly affect functioning and quality of life. Indeed, SMD represent a major contributor to the total amount of disability-adjusted life-years attributed to communicable and non-communicable diseases at a global level (5). This appears to be mainly determined by the third determinant of burden, i.e., the presence of suboptimal patterns of response to treatments, either pharmacological or nonpharmacological, leading to only a minority of patients achieving psychopathological and functional remission. Finally, SMD are associated with a considerable excess morbidity and mortality (6-8), which cause a significant reduction in life expectancy (on average 10-20 years) compared to the general population $(9,10)$. In this context, there has been a constant attempt to improve outcomes of SMD. This strategy has mainly focused on prevention, with the most validated paradigm focusing on primary prevention in individuals presenting subtle symptoms and at clinical high risk for SMD (11). Although the early phases of SMD appear to have distinct developmental trajectories for major affective disorders (4) and schizophrenia (3), particularly in the prodromal phases, there is a general consensus that individuals at risk for SMD are those having a genetic liability due to a high familial loading and/or the presence of antecedents such as basic symptoms, cognitive development, affective lability, anxiety, sleep problems, and psychotic-like experiences $(11-13)$.

In this context, risk prediction of SMD is of paramount importance. Several modeling approaches have been developed using clinical (phenotypic) (14), genomic $(15,16)$, epigenomic (17), or integrated phenotypic-omics datasets (18). However, although the accuracy of prediction in the proposed models appears adequate for clinical purposes (18), and/or feasible in their implementations (14), there is still need of replication and validation of their predictive power in real life clinical settings. One biological component, partly inherited (19), that has been so far neglected in risk prediction of SMD, is the microbiota. The human microbiota consists in the ensemble of microbes, including viruses, bacteria, and eukaryotes, that inhabit several ecological niches of the organism $(20,21)$. Due to its demonstrated role in modulating illness and health, much interest has focused on the characterization of the microbiota inhabiting the gut (20). In fact, alterations of the gut microbiota have been linked, among the others, to obesity (22), maturation of the immune system (23), and response to drugs (24). Of particular interest is the modulating role that the microbiota acquires in human behavior (25), raising the interest for the investigation of its modifications in SMD. Indeed, several studies have shown substantial alterations, mainly decreased diversity in species within the microbiota, in schizophrenia $(26,27)$, in bipolar disorder (28), and major depressive disorder (29, 30). For instance, Zhu and coauthors found that, compared to 81 healthy controls, the gut microbiota of 90 medication-free patients with schizophrenia harbored many facultative anaerobes such as Lactobacillus fermentum, Enterococcus faecium, Alkaliphilus oremlandii, and Cronobacter sakazakii/turicensis, typically rare in a healthy gut (31). Of note the schizophrenia-associated bacterium Streptococcus vestibularis, which contributed to the microbiota metagenomic-based discrimination of patients with schizophrenia from healthy controls, when transplanted to mice gut induced deficits in social behaviors, altering neurotransmitter levels in peripheral tissues of recipient animals (31). In bipolar disorder, Painold and co-authors found that gut microbiota alpha-diversity decreased with increasing illness duration and that Actinobacteria and Coriobacteria were overrepresented in patients compared to healthy controls (HC) (28). Finally, patients with major depressive disorder showed a statistically significant overrepresentation of Bacteroides enterotype 2 compared to controls (32). In addition, a recent systematic review showed that gut dysbiosis and the leaky gut may affect pathways implicated in the neurobiology of major depressive disorder, such immune regulation, oxidative and nitrosative stress, and neuroplasticity (29). However, there is still limited evidence on how microbiota might vary in individuals at risk for SMD compared to healthy controls, as well as to individuals in later stages of SMD. However, there is extensive evidence that the microbiota has a key role in neurodevelopment and can be a modulating factor of the maturity of the central nervous system (CNS) in early developmental stages (33). In this scenario, the aim of this mini review is to present the current evidence on microbiota changes in individuals at high risk for SMD, offering a developmental perspective and providing mechanistic insights on how changes in the gut microbiota make-up could influence the risk of SMD.

\section{GUT MICROBIOTA IN AT RISK MENTAL STATES: A DEVELOPMENTAL PERSPECTIVE}

Recent evidence suggests that the shaping of the microbiome occurs in parallel with the growth of CNS and that they have similar critical developmental windows (34). Consequently, the influence of alterations of gut microbiota on brain maturation trajectories, as well as their relationship with an increased risk for mental disorders later in life have been extensively investigated by preclinical studies $(35,36)$. In fact, alterations in maternal microbiome have been shown to impact offspring's brain maturation and post-natal development of psychopathology. Buffington et al. (37), observed that the offspring of high-fat diet exposed mice showed autism spectrum disorders/schizophrenia-like symptoms, such as reduced social interactions, poor interest in social novelty, and altered sociability compared to the offspring of normal fed mice (37). These behavioral alterations were coupled with a 9fold reduction of Lactobacillus reuteri and a reduced number of cells producing oxytocin in the paraventricular nuclei of the hypothalamus (37). Other studies investigated the effect of altered maternal gut microbiome on the offspring's behavior through the administration of antibiotics during or 
immediately before mice pregnancy. A plethora of postnatal aberrant behavior, such as decreased locomotor and explorative activity, low prepulse inhibition, poor social interactions, and anxiety emerged $(38,39)$. Interestingly, aberrant behavior was completely reversed after fostering the pups by control dams (39). Other factors, such as maternal exposure to stress, can alter the offspring's gut microbiome and affect behavior. Several studies showed that the offspring exposed to perinatal maternal stress showed decreased levels of Lactobacillus and Bifidobacterium (40-42). These alterations were associated to increased anxiety and impaired cognitive functions, which started early during development and lasted until adulthood (4042). Furthermore, gut microbiome composition and behavioral alterations were paired with increased levels of interleukin- $1 \beta$ and decreased brain-derived neurotropic factor (BDNF) in the amygdala (41).

Together with the intrauterine stage, the postnatal period represents a critical moment for both gut microbiota and brain development (34). This developmental stage represents the time when the most dramatic changes in the composition of the intestinal microbiota take place. These are mainly driven by a series of factors, spanning from maternal delivery modalities to genetic diathesis (43-45). Therefore, the interactions between the developing gut microbiota and brain structure and function in this specific developmental phase have undergone extensive investigations. Sudo et al. reported that germfree (GF) mice, i.e., animals that have never had contact with any microorganism, showed heightened hypothalamicpituitary-adrenal (HPA) system response to acute restraint stress as compared to mice with a normal gut flora (46). Such phenotype was accompanied by reduced expression of hippocampal and cortical brain-derived neurotrophic factor (BDNF). When GF were administered with a single strain of bacterium, Bifidobacterium infantis, stress response normalized (46). However, normalization processes were only possible in GF at early developmental stage, whereas the same procedure in later stages had no effects (46). Another study (47) demonstrated that GF mice showed anxious behavior and increased levels of serotonin in the hippocampus. Even in this case, gut colonization after weaning, which is comparable to adolescence in humans, was uncapable of restoring normal serotonin levels, even though anxiety normalized. Accordingly, in another study (48), post-weaning bacteria colonization was not able to normalize myelin oligodendrocyte glycoprotein levels in GF mice. Cumulatively, these data point toward the existence of specific, and limited, critical periods for the gut microbiota to act on neuronal circuits function and plasticity. The work of Desbonnet et al. (49) further expanded such concept. In their work, post-weaning colonization only partially corrected autism-spectrum-disorder-like behavior in GF mice: self-grooming and social avoidance improved, whereas social cognition did not (49). The authors suggested that the window of opportunity for the microbiota to impact brain circuits might be different for distinct emotional/social behaviors and, eventually, sensory modalities (49). These findings are summarized in Table $\mathbf{1}$.

\section{GUT MICROBIOTA IN AT RISK MENTAL STATES: CLINICAL DATA}

Despite the relatively large amount of studies investigating the relationship between gut microbiota composition and neurodevelopmental alterations in mice, only one study investigated the effect of altered microbiome and risk of developing mental disorders in humans (51). Specifically, $\mathrm{He}$ et al. (51) investigated alpha-diversity (i.e., the bacterial diversity within a single sample) and beta-diversity (differences in species composition among samples) metrics of gut microbiome in high-risk (HR), ultra-high-risk (UHR) subjects for developing schizophrenia and HC (51). Beta-diversity analysis revealed that UHR and HR had significantly different global microbiome composition than HC. Furthermore, UHR showed greater levels of Clostridiales, Lactobacillales, Bacteroidales, higher levels of Acetyl coenzyme A synthesis and greater anterior cingulate choline levels than the both HR and HC. The authors pointed out that the alterations in microbiome overlapped with those identified in schizophrenia and autism-spectrum disorder $(52,53)$. Additionally, higher levels of choline were interpreted as resultant of altered membrane metabolism due to microglial activation, which is one of the possible mechanisms mediating the effects of an altered gut microbiome on neural development (51). Putative mechanisms of the interplay between microbiota and genetic predisposition in modulating the liability toward the development of a SMD is discussed below. We have summarized clinical evidence in Table 2.

\section{MECHANISTIC HYPOTHESES ON THE INFLUENCE OF GUT MICROBIOTA ON AT RISK STATUS FOR SEVERE MENTAL DISORDERS}

There is compelling evidence that the products of gut microbiota might influence behavior in mammals through the action of their byproducts on the CNS (25). For instance, metabolic waste products of the gut microbiota such as the shortchain fatty acids (SCFAs) can influence neuromodulation via inhibition of the histone deacetylases $(25,54)$. In addition, another byproduct such as butyrate helps maintaining the integrity of the blood-brain barrier $(25,55)$, while acetate appears to exert anorectic effects via preferential accumulation in the hypothalamus (56). Other sets of findings have pointed to the link between gut dysbiosis and increased gut permeability and alterations of mitochondrial function, with significant repercussions at the CNS level (57). This amount of evidence, supported by the clinical and preclinical findings on the impact of gut microbiota on neurodevelopment, has fostered several mechanistic hypotheses $(58,59)$. While an extensive discussion of these mechanisms is out of the scope of the present mini review, we present a synthesis that we reckon as relevant for the high-risk construct of SMD. An altered neurodevelopment due to maternal gut flora modifications might be the resultant of poor regulation of maternal/fetus inflammatory state mediated by the maternal 
TABLE 1 | Summary of findings of the pre-clinical studies and/or postulated biological underpinnings of SMD on gut microbiota.

Animals $\quad$ Tested hypothesis Preclinical model $\quad$ Tested biological correlates $\quad$ Findings/results

GF, SOF BALC/C mice Microbiome

influence on the

Acute restraint stress, ether stress response (N/A)

GF, SPF NMRI (males, $8-10$ weeks old)

Gut microbiome influence on norma brain development and behavior (N/A)

GF, CC Swiss Webster Sex differences in (males and females, 6-9 the gut microbiome weeks old)

\section{regulation of the} hippocampal serotonergic system (N/A)

GF, CC Swiss Webster (miluence on social NIH Swiss strain as stimulus mice in the tests

BALB/C mice VPA-E and CON VPA-E mice were exposed in utero at G11; males and The association between altered gut microbiota and autism-like
Open field test, Light-Dark Box test, Elevated Plus Maze test

Novel-environment stress T; 5-HIAA, 5-HT, KYNA assessed through HPLC; TNF- $\alpha$

following LPS splenocyte stimulation;

Plasma ACTH, CRT, IL-1 $\beta$ and IL-6 microbiota through culture; RT-PCR $\mathrm{CRH}, \mathrm{GR}, \mathrm{NMDAR}$ gene expression on CTX, HPC, HPT; ELISA for BDNF, NT-3, NGF on HPC and HPT.

NA, MHPG, DA, DOPAC, HVA, 5-HT, and 5-HIAA on CTX, HPC, STR tissue assessed through RPHPLC;

assessment of the NGFI, BDNF, DR1, DR2, DARPP-32 expression with ISH on AMG, HPC, CTX samples; SNP. PSD-95 assessed through WB on CTX STR, HPC.

Sociability and social novelty N/A preference (three chamber test); social transmission of food preference test

Social behavior scores (time Cecal levels of SCFA (i.e. acetic spent near unfamilia gendermatched mouse) propionic, butyr

and valeric acids); 16S rRNA analysis on cecal samples to investigate the stool bacteria composition
Higher ACTH and corticosterone plasma levels in response to restraint stress among GF mice as compared to SPF, but not in response to ether stimulation. Lower BDNF expression among GF in CTX and HPC tissues as compared to SPF. Normalization of the HPA stress response with an early reconstitution of the gut microbiome with Bifidobacterium infantis; an ncreased stress response was observed with enteropathogenic Escherichia coli, but not with a strain devoid of the translocated ITR gene.

GF showed altered expression of genes involved in second messenger pathway and synaptic potentiation, as well as increased motor activity and lower anxiety behaviors as compared to SPF. Early exposure of GF to gut microbiota resulted in a normalization of GF locomotor activity; adult

exposure to gut microbiota failed to normalize GF behaviors. GF presented higher expression of SNP, PSD-95 in the STR as compared to SPF. Higher turnover rates were observed among GF for NA, DA, 5-HT in STR as compared to SPF. GF subjects presented lower BDNF expression in the HPC, AMG, CTX, and lower expression of NGFI-A in the STR, CTX, HPC compared to SPF.

GF presented a lower TNF- $\alpha$ production following LPS stimulation and a higher CRT response to stress as compared to CC regardless of gender male GF subjects presented lower BDNF, and higher production of 5-HT and 5-HIAA in the HPC as compared to CC, as well as a higher plasma TRP and a decreased KYNA/TRP ratio; GF female had a lower body weight as compared to CC. Gut microbiota recolonization led to a normalization of TRP concentration and of anxiety-like behaviors; no effect was described on the 5-HT and 5-HIAA concentrations in the HPC.

- Social impairment among GF as compared to CC (i.e., more time spent in an empty chamber instead of one shared with another subject); GF did not spend more time analyzing unfamiliar environment over familiar ones, as compared to CC.

A second cohort confirmed social deficits and reduced preference for social novelty among GF; the post-weaning bacterial colonization resulted in the reversal of the observed social aversion but did not affect social cognition impairments. GF spent more time in repetitive self-grooming behaviors and less time in social investigation during the socia transmission of food preference test; these behaviors normalized after gut microbiota colonization.

Butyric acid levels were higher among male VPA-E mice as compared to CON. No difference was found for the other SCFA assessed. OTU expression was significantly influenced among VPA-E males; changes observed in the gut microbiota correlated with increased ileal neutrophil infiltration, increased intestinal butyrate level, a reduced level of intestinal serotonin and lower social behaviors score. 


\section{Animals} (gender, age)

Tested (SMD)

SD rats, PNS-E and CON (PNS rats were exposed to restraint stress during gestational day 14-20; males, 2-4 months old)

The complex interplay between prenatal stress,

major physiologica systems and gut

microbiota

composition

Wistar rats, SST-E and CON (SST-E rats were exposed to an

unabsorbable antibiotic starting 1 month before breeding until gestational day 15; male and female, 3-7 weeks old)

C57/B16 mice, PNS-E and CON were exposed during gestational day 10-16; females, 8-10 weeks old)
GF and CC Swiss
Webster (males and
females, 10 weeks old

\section{Gut microbiota} influence on prefrontal cortex myelination

The complex interplay between perinatal antibiotic exposure and the health

The complex interplay between perinatal stress, commensal microbes and anxiety-like behaviors in the female offspring
Tested biological correlates

Findings/results

References

Behavioral screening (Op

novel object recognition);

acute restraint test

1st cohort: colon excision and analyzed for innervation density

(confocal fluorescence imaging), and secretomotory function (chambers)

- 2nd cohort: 16S rRNA analysis on fecal samples to investigate the stool bacteria composition; tail-bleed plasma corticosterone assessment following acute restraint test, somatic pain sensitivity with the hot plate test and respiratory function (whole body plethysmography)

- 3rd cohort: blood pressure, colorectal distension, acute restraint stress

Behavioral screening (open Homocysteine and tryptophan levels field, social interactions, among untested siblings and dams marble burying, elevated (preconceptional and post euthanasia plus maze, prepulse inhibition of the acoustic startle reflex)

No abnormality was documented in the homocysteine and tryptophan levels between SST-E and CON, ruling out folate deficiency in the SST-E group. SST-E showed decreased social interactions, increased anxiety behaviors (i.e., reduced exploration of the open arm in the elevated plus maze), and altered sensorimotor gating (i.e., reduction in the startle inhibition)

PNS-exposure resulted in a decreased distal colon innervation and an increased secretory response to cathecholaminergic stimulation;

PNS-exposed rats presented a lower expression of Lactobacillus and a observed changes in the gut microbiota correlated with respiratory and HPA-axis changes.
Behavioral screening (elevated plus maze, nove object recognition test, tal suspension test)
- 1st cohort: euthanized at the 17 th gestational day for tissue collection

- 2nd cohort: behavioral testing, parturition, microbiome sampling (16S rRNA), tissue collection from offspring (i.e., IL-1 $\beta, \mathrm{BDNF}$ in placental, and in both fetal and maternal brain)

RNA-sequencing, qRT-PCR within various brain regions to investigate myelin component genes; protein extraction and western blot; transmission electron microscopy on prefrontal cortex samples (gathered from 6 male mice)
Stress exposure influenced the maternal gut microbiota; no significant difference was found in the placental microbiota composition. PNS-E mice presented higher Bacteroides and Firmicutes expression as compared to CON; at the family level, a relative increase of the Bifidobacteriaceae, and Rlkenellaceae was described. Prenatal stress exposure resulted in increased anxiety-like behaviors and neophobia. Stress exposure resulted in reduced BDNF placental levels and higher levels of IL-1 $\beta$ in placental and fetal brain tissues; adult PNS-E mice had lower BDNF levels in the amygdala.

Differential expression of 250 genes between GF/colonized-GF and CON (14 out of the 94 upregulated genes in GF were directly involved in myelinization, but none of them were upregulated in the colonized-GF); GF and colonized-GF differed for the expression of 15 genes. QRT-PCR confirmed abnormal expression of five myelin component genes among GF; the increased in mRNA expression was confined to the prefrontal cortex, and the gut colonization resulted in the normalization of the genes expression. Electron microscopy revealed increased myelinization among GF regardless of axonal diameter; normalization of mRNA transcription with colonization did not result in a reduction in the relative myelin protein abundance. 


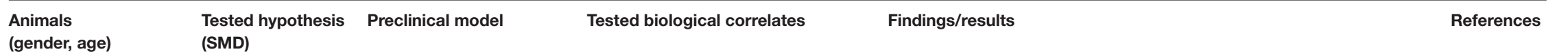

(gender, age)

(SMD)

SPF C57BL/6 mice UAA-E and CON (UUA mice were exposed to an unabsorbable antibiotic in utero during gestational day 9-16; males and females, 4 weeks old)

The effects of Open field test, social behaviors chambers social test), $24 \mathrm{~h}$ home cage activity test.

Study subjects divided in 3 cohorts:

- 1st cohort: exposed to

UAA in utero and fostered by CON

2nd cohort: not exposed

to UAA in utero and fostered by CON

- 3rd cohort: not exposed

to UAA in utero and

fostered by CON

SPF C57BL/6, GF, ASF (males and females,

6-10 weeks old)
Investigating the role N/A

of gut microbiota in

microglia maturation

process
$16 \mathrm{~S}$ rRNA analysis on fecal samples to investigate the stool bacteria composition exposed dams and control dams; different expression of gut microbiota was reported between UAA and CON groups also in the offspring. UAA offspring presented lower body weight, lower activity levels in the dark phase of the $24 \mathrm{~h}$ home cage activity test, reduced locomotor activity in the open field test, and reduced rearing behaviors in a novel environment. The 1 st and the 2nd cohort presented a similar phenotype at week 4 and differed significantly from the 3rd cohort.
16S rRNA analysis on fecal samples to investigate the stool bacteria composition; LMCV challenge through right hemisphere injection; LPS challenge applied intracerebrally and intraperitoneally under anesthesia; RT-PCR on adequately processed FACS-separated microglial cells to analyze gene expression; histology $\mathrm{IHC}$ and three-dimensional microglia reconstruction
Different mRNA expression between SPF and GF mice was observed especially among genes involved in cell activation, in pathogen recognition consistent with immature microglia phenotype. GF presented more lba-1+ microglial cells featuring longer processes, more segments and establishing more physical contacts with adjacent cells as compared to SPF. LPS challenge and LCMV test revealed an abnormal immune response among GF subjects, heralded by differential expression of genes involved in the immune response and prominent morphological anomalies. Antibiotic exposure for 4 weeks, induced similar phenotypic changes in microglial cells among SPF, but with no changes in cell numbers; ASF tri-colonized (Bacteroides distasonis, Lactobacillus salivarius, Clostridium cluster) presented both increased microglial cell numbers and morphological changes, despite having near-normal biomass, reversible by allowing a more diverse bacterial colonization with SPF co-housing. Intriguingly, a 4-week course of SCFA supplements resulted in the normalization of the microglial phenotype among GF.

*/L-6 bioactivity assessed through IL-6-dependent $B$ cell hybridoma.

5-HIAA, 5-Hydroxyindoleacetic acid; 5-HT, 5-Hydroxytryptamine; ACTH, Adrenocorticotropic hormone; AMG, Amygdala; ASF, Altered Schaedler flora; BDNF, Brain Derived Neurotrophic Factor; CC, Conventionally Colonized; CRH, Corticotropic Releasing Hormone; CON. Controls; CRT. Corticosterone; CTX. Cortex; GF, Germ Free; DA, Dopamine; DARPP-32, Dopamine- and cAMP-regulated phosphoprotein Mr 32 kDa; DOPAC, Dihydroxyphenylacetic acid; DR1, Dopamine Receptor 1: DR2, Dopamine Receptor 2; G11, Gestational day 11th; GF, Germ Free; GR, Glucocorticoid Receptor; HPA, Hypothalamic Axis; HPC, Hippocampus; HPLC, High-Performance Liquid Chromatography; HPT, Hypothalamus; HVA, Homovanillic acid; IHC, Immunohistochemistry; ISHT, In Situ Hybridation; ITR, Intimin Receptor; KYNA, Kynurenic acid; LCMV, Lymphocytic Choriomeningitis Virus; LPS, LipoPolySaccharide; MHPG, 3-Methoxy-4HydroxyPhenyIGlycol; N/A, Not Available; NA, noradrenaline; NGFI-A, Nerve Growth Factor-Inducible clone A; NMDAR, N-Methyl-D - Aspartic Acid Receptor subunits (NR-1 and NR-2A); NGF, Nerve Growth Factor; NT-3, Neurotrophin-3; OTU, Operational taxonomic unit; PNS-E, Prenatal stress in utero exposure; PSD - 95, Postsynaptic density protein 95; qRT-PCR, quantitative Real Time Polymerase Chain Reaction; RPHPLC, Reversed-Phase High-Performance Liquid Chromatography; SCFA, Short chain fatty acids; SD, Sprague-Dawley; SMD, Severe Mental Disorder; SNP, Synaptophysin; SPF, Specific Pathogen Free; SST-E, Succinyl Sulfa Thiazole exposed in utero; STR, Striatum; TNF- $\alpha$, Tumor Necrosis Factor- $\alpha$; T-RFLP, Terminal Restriction Fragment Length Polymorphism; UAA, Unabsorbable Antibiotic; UAA-E, Unabsorbable Antibiotic exposure in utero; VPA-E, Valproic Acid in utero Exposure; WB, Western Blotting. 


\section{SMD (diagnostic Sample size and composition}

criteria)

AD, PDD (DSM - $\quad \mathrm{AD} n=10, \mathrm{PDD}-\mathrm{NOS} n=10, \mathrm{HC} n \quad$ Cross-sectional study; sacteria composition, its metabolic

HR, UHR (DSM - HR $n=81 ; \mathrm{UHR} n=19 ; \mathrm{HC} n=69$ Cross-sectional study;

IV) (13-30 y.0.; HR 41 M, 40F; UHR $15 \mathrm{M}, 4 \mathrm{~F} ; \mathrm{HC} 37 \mathrm{M}, 32 \mathrm{~F}$

SCZ (ICD-10) SCZ $n=64, \mathrm{HC} n=53$ (18-65 y.o.; Cross-sectional study; $16 \mathrm{~S}$ rDNA and 16S $36 \mathrm{M}, 28 \mathrm{~F}$ in SCZ; $35 \mathrm{M}, 18 \mathrm{~F}$ in $\mathrm{HC}) \quad$ rRNA analysis on fecal samples to investigate

GP reported depression (NA)

Subset of the FGFP cohort $n=1,054$ Cross-sectional study; BMI; BSS; GP reported sets $n=1,070$

$=7$ group.

m.a. 57.9 y.o., $447 \mathrm{M}, 616 \mathrm{~F}$; TR-MDD balanced to the FGFP group)

Bipolar Disorder $\quad \mathrm{BD} n=32 ; \mathrm{HC} n=10$

(DSM-IV) (BD 20-65 y.o., 18 M, 14 F; HC NA y.o., $4 \mathrm{M}, 6 \mathrm{~F})$
Cross-sectional study; BDI-II; HAM-D; inflammatory markers, serum lipids, KYNA oxidative stress and anthropometric measures: $16 \mathrm{~S}$ rRNA analysis on fecal samples to investigate the stool bacteria composition.

Schizophrenia $90 \mathrm{SCZ}, 81 \mathrm{HC}$, validated in a Cross-sectional; MWAS to characterize gut (DSM-IV)

verification sample $45 \mathrm{SCZ}^{1}$ and 45 $18-64$ y.0.,41 M, 40F) microbiota; MCCB; PANSS; KYNA and

probe mice stool levels; 16 S rRNA analysis t (the activity and an assessment of the organic volatile compounds and free fatty acids composition.

1H-MRS; APSS, BIPS, GAF-M, GRDS, SIPS, SOPS; HR and UHR were screened for the absence of DSM - IV coded diagnoses; $16 S$ rRNA analysis on fecal samples to investigate the stool bacteria composition. the stool bacteria composition; PICRUS analysis to probe metabolic pathways; PANSS.

PDD-NOS and HC presented higher Faecalibacterium and

Ruminococcus expression; PDD-NOS and HC presented higher expression of Caloramator, Sarcina, and Clostridium; PDD-NOS and AD presented different composition of Lachnospiraceae as compared with the HC. Different levels of organic volatile compounds and free fatty acid between the three groups.

Increased expression of Clostridiales, Lactobacillales and Bacteroidales in UHR compared to the other two groups; increased choline levels on $1 \mathrm{H}-\mathrm{MRS}$ among UHR subjects compared to the other groups.

SCZ patients presented higher expression of the Proteobacteria phylum, and at the genus level, a relatively higher expression of Succinivibrio, Megasphaera, Collinsella, Clostridium, Klebsiella,

Methanobrevibacter, and a lower of Blautia, Coprococcus, Roseburia as compared to HC; differences in numerous metabolic pathways between $\mathrm{HC}$ and SCZ (e.g. fatty acid, vitamin B6).

Butyrate-producing

Faecalibacterium and Coprococcus bacteria were associated with higher QOL. Dialister, Coprococcus spp. depletion was observed in depression; microbial synthesis of 3,4-dihydroxyphenylacetic acid appeared positively correlated with mental QOL. analysis on fecal samples to investigate the stool bacteria composition.
(FGFP m.a. 50.9, 478M, 576 F; LLD
BD illness duration was negatively correlated with microbial alpha diversity. Actinobacteria and

Coriobacteria were more abundant in $\mathrm{BD}$ as

compared with HC; Ruminococcaceae and Faecalibacterium

were more abundant in $\mathrm{HC}$ as compared with $\mathrm{BD}$. Certain bacteria

clades were more commonly observed with the metabolic and inflammatory patterns

observed

among $\mathrm{BD}$ individuals.

Different tryptophan and KYNA blood levels between SCZ and HC; SCZ gut microbiota featured higher expression of

facultative

anaerobes and oral cavity bacteria as compared with HC. Transplantation of Streptococcus vestibularis in mice resulted in altered neurotransmitter production and social behaviors.
(52)

*TR-MDD: TR-MDD was defined as a diagnosis of either Major Depressive Disorder or Bipolar Type Il according to the DSM-IV criteria. 1H-MRS, Proton Magnetic Resonance Spectroscopy; AD, Autism Disorder; ADI-R, Autism Diagnostic Interview-Revised; ADOS, Autistic Diagnostic Observation Schedule; APSS, Attenuated Positive Symptom Syndrome; BIPS, Brief Intermittent Psychotic Syndrome; BDI-II, Beck Depression Inventory; BMI, Body Mass Index; BSS, Bristol stool scale; DSM - IV, Diagnostic and Statistical Manual of Mental Disorders IV edition; F, Female; FDO, Free Direct Observation; FGFP, Flemish Gut Flora Project; GAF-M, General Assessment of Functioning - Modified version; GP, General Practitioner; GPRD, General Practitioner Reported Depression; GRDS, Genetic Risk and Deterioration Syndrome; HAM-D, Hamilton Depression Rating Scale; HC, Healthy Control; KYNA, Kynurenic Acid; LLD, Dutch LifeLines DEEP; M, Male; m.a., mean age; MCCB, MATRICS Consensus Cognitive Battery; MWAS, Metagenome-Wide Association Study; NA, Not Available; $n$, total size; PANSS, Positive and Negative Syndrome Scale; PDD-NOS, Pervasive Developmental Disorder - Not Othenwise Specified: PICRUSt, Phylogenetic Investigation of Communities by Reconstruction of Unobserved States: QOL, Quality Of Life: RAND-36, RAND-36 health-related quality of life survey; SCZ,

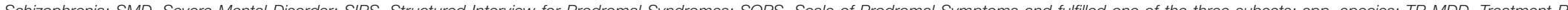
Depressive Disorder; y.o., years old. 


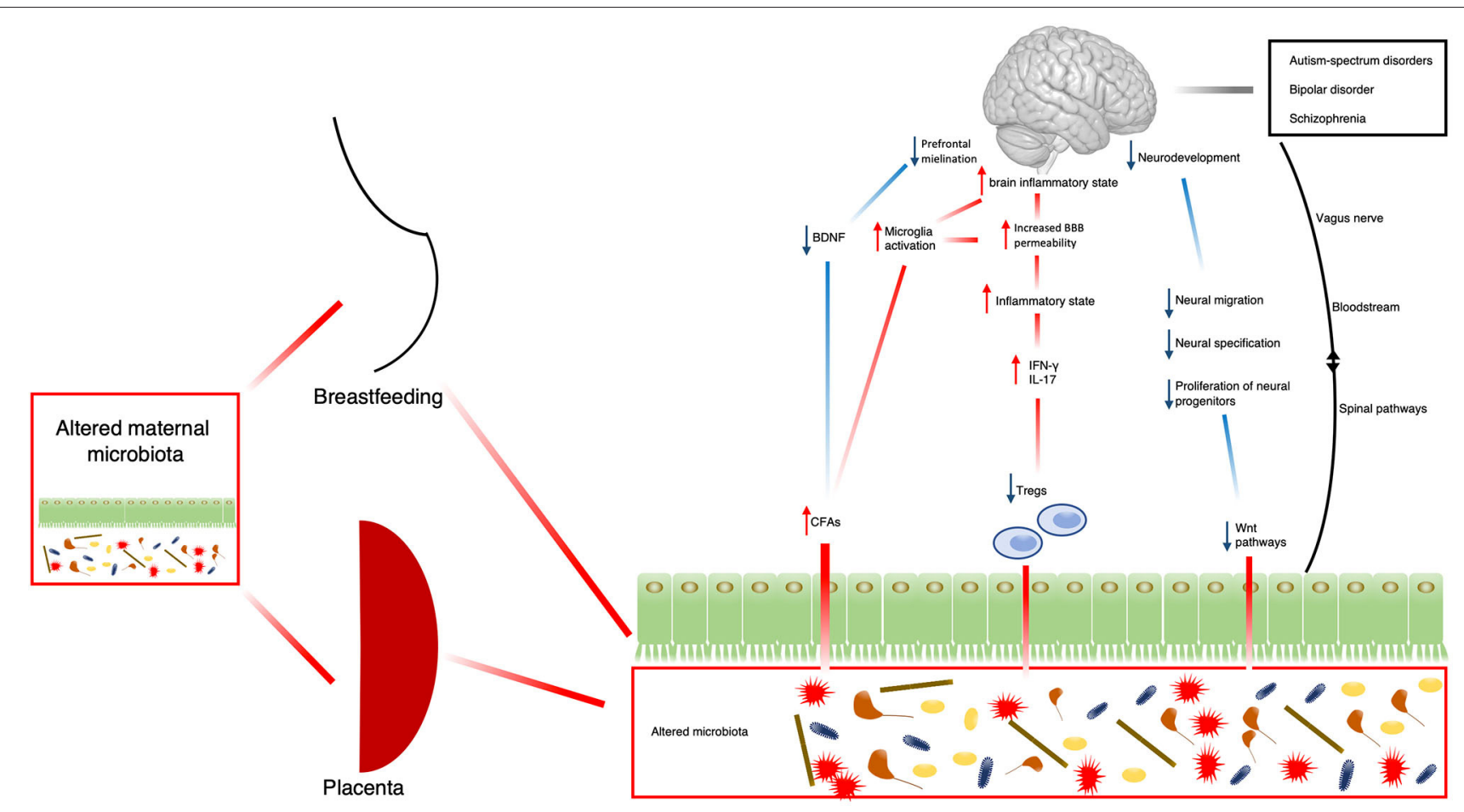

FIGURE 1 | Schematic representation of possible mechanisms by which the microbiota might contribute to the development of SMD. BDNF, brain-derived neurotrophic factor; CFAs, fermentation products; IFN- $\gamma$, interferon- $\gamma$; IL-17, Interleukin-17; Tregs, regulatory T-cells; Wnt, homologous wingless and Int-1.

gut microbiome (58). Adequate gut microbial colonization in pregnant mice was associated to expression of regulatory $\mathrm{T}$ cells (Tregs). Tregs normalize systemic levels of proinflammatory cytokines, such as IL-17 and interferon- $\gamma(60)$, thus maintaining correct inflammatory/non-inflammatory balance. The lack of gut microbiota in GF pregnant mice resulted in a decrease of Tregs, with a general imbalance toward maternal and fetal inflammatory state (60). High levels of proinflammatory cytokines have been shown to induce fetal abnormal cortical development and surge of post-natal autism-like behavior (61). Alteration of maternal gut microbiome might also increase levels of fermentation products (CFAs), namely acetate, propionate and butyrate (62). Indeed, CFAs are capable to massively activate microglia, the immune cells of the CNS playing an important role in CNS homeostasis (50). Microglia activity might initiate/exacerbate the inflammatory cascade leading to the massive release of cytokines as well as to associated alterations in the endothelial permeability, including the blood-brain barrier. Such cascade has been shown to predispose to the development of neurodegenerative disorders, including schizophrenia and Parkinson's disease $(59,63)$.

Another putative mechanism might involve alterations in neurogenesis and specifically the BDNF which is involved in neural growth and cell survival. As previously shown, the gut microbiota is involved in the expression of BDNF (64). Prenatal/postnatal alterations of the gut microbiota can alter BDNF expression, and these changes could alter maturation trajectories of neural circuitry, leading to the development of SMD (65-68). Furthermore, gut microbiota can modify oligodendrocyte products and affect myelination, particularly in the prefrontal cortex, a brain region involved in attention, memory, emotional learning and critically connected to SMD such as ASD (69), schizophrenia (70), major depressive disorder (71), bipolar disorder (72), and substance abuse (73). Specifically, altered myelination has been related to changes in synaptic formation and function, which could lead to the surge of specific cognitive deficits typically seen in schizophrenia, namely deficits in attention, working memory, and executive function (74).

Another interesting, but still under-investigated, mechanism is represented by the effect of the gut microbiome on the Wnt pathways. These are signal transduction pathways mainly involved in human development, cell migration and proliferation and tissue regeneration (75). Wnt pathways are also involved in neural morphogenesis, axon guidance, neurite outgrowth, and synaptic plasticity $(76,77)$. Alterations in Wnt pathways have been recently related to higher risk to develop SMD, such as schizophrenia or bipolar disorder $(78,79)$. Of note, GF mice showed poor Wnt pathway activity in intestinal stem cells (80), supporting the speculation of a possible link between alteration of gut microglia, altered neurodevelopment and consequent increased risk for SMD. However, proper investigation of the relationship between gut microbiome alterations and altered Wnt pathways is still underdeveloped and needs further research. All these mechanisms are illustrated in Figure 1. 


\section{CONCLUSIONS}

The findings of our review prompt a series of considerations. First, despite the consensus that microbiota plays a fundamental role in neurodevelopment and substantial changes are detectable in individuals affected by SMD, there is a dearth of studies investigating its modifications during the developmental trajectories of these disorders, particularly in high-risk populations. This could be feasible particularly in consideration that appropriate clinical chemistry and molecular immunology assays to assess for the presence of biological markers of "leaky gut" might be easily implementable in clinical settings (81). Second, only a longitudinal perspective could shed light on the direction of these changes, i.e., whether microbiota modifications precede the onset of psychopathology (of whatever severity) or vice versa. This perspective could be applied, but should not be limited, to the early stages of SMD. Indeed, prospective analysis of microbiota changes are starting to shed light on the longitudinal variation of mood in the course of bipolar disorder (82). Third, this approach can help decrease the confounding associated with the use of drug treatments (if the analyses are performed in pre-diagnostic stages), and at the same time inform on changes that might favor, or be predictive of, response to treatment. In conclusion, we suggest that the analysis of

\section{REFERENCES}

1. Rehm J, Shield KD. Global burden of disease and the impact of mental and addictive disorders. Curr Psychiatr Rep. (2019) 21:10. doi: 10.1007/s11920-019-0997-0

2. Gustavsson A, Svensson M, Jacobi F, Allgulander C, Alonso J, Beghi E, et al. Cost of disorders of the brain in Europe 2010. Eur Neuropsychopharmacol. (2011) 21:718-79. doi: 10.1016/j.euroneuro.2011.08.008

3. Millan MJ, Andrieux A, Bartzokis G, Cadenhead K, Dazzan P, Fusar-Poli P, et al. Altering the course of schizophrenia: progress and perspectives. Nat Rev Drug Discov. (2016) 15:485-515. doi: 10.1038/nrd.2016.28

4. Duffy A, Goodday S, Keown-Stoneman C, Grof P. The emergent course of bipolar disorder: observations over two decades from the Canadian high-risk offspring cohort. Am J Psychiatr. (2019) 176:720-9. doi: 10.1176/appi.ajp.2018.18040461

5. Hmwe Kyu H, Abate D, Hassen Abate K, Abay SM, Abbafati C, Abbasi $\mathrm{N}$, et al. 2018 Global, regional, and national disability-adjusted lifeyears (DALYs) for 359 diseases and injuries and healthy life expectancy (HALE) for 195 countries and territories, 1990â€“2017: a systematic analysis for the global burden of disease study 2017. Lancet. 392:1859-922. doi: 10.1016/S0140-6736(18)32335-3

6. Harris C, Barraclough B. Excess mortality of mental disorder. Br J Psychiatr. (1998) 173:11-53. doi: 10.1192/bjp.173.1.11

7. De Hert M, Correll CU, Bobes J, Cetkovich-Bakmas M, Cohen D, Asai I, et al. Physical illness in patients with severe mental disorders. I Prevalence, impact of medications and disparities in health care. World Psychiatr. (2011) 10:52-77. doi: 10.1002/j.2051-5545.2011.tb00014.x

8. Chesney E, Goodwin GM, Fazel S. Risks of all-cause and suicide mortality in mental disorders: a meta-review. World Psychiatr. (2014) 13:153-60. doi: 10.1002/wps. 20128

9. Druss BG, Zhao L, Von Esenwein S, Morrato EH, Marcus SC. Understanding excess mortality in persons with mental illness: 17-year follow up of a nationally representative US survey. Med Care. (2011) 49:599-604. doi: 10.1097/MLR.0b013e31820bf86e

10. Nordentoft M, Wahlbeck K, Hällgren J, Westman J, Ösby U, Alinaghizadeh H, et al. Excess mortality, causes of death and life expectancy in 270,770 patients microbiota should be included in the comprehensive assessment generally performed in populations at high risk for SMD as it can inform predictive models and ultimately preventative strategies.

\section{AUTHOR CONTRIBUTIONS}

MM and GS drafted the first version of the article. AS, DJ, and PP helped with the search of the literature and contributed to the draft of the manuscript. FP and BC oversaw the project and revised the text critically for important intellectual content. All authors gave final approval of the version to be published and agree to be accountable for all aspects of the work.

\section{FUNDING}

This paper was partly funded by Fondo Integrativo per la Ricerca (FIR)-2019 granted to MM, FP, and BC.

\section{ACKNOWLEDGMENTS}

The authors wish to thank all patients affected by severe mental illness who make our research possible, and most importantly meaningful.

with recent onset of mental disorders in Denmark, Finland and Sweden. PLoS ONE. (2013) 8:e0055176. doi: 10.1371/journal.pone.0055176

11. Fusar-Poli P, Borgwardt S, Bechdolf A, Addington J, RiecherRössler A, Schultze-Lutter F, et al. The psychosis high-risk state: A comprehensive state-of-the-art review. Arch Gen Psychiatr. (2013) 70:107-20. doi: 10.1001/jamapsychiatry.2013.269

12. Zwicker A, MacKenzie LE, Drobinin V, Howes Vallis E, Patterson VC, Stephens $\mathrm{M}$, et al. Basic symptoms in offspring of parents with mood and psychotic disorders. BJPsych Open. (2019) 5:e54. doi: 10.1192/bjo.2019.40

13. Zwicker A, Drobinin V, MacKenzie LE, Howes Vallis E, Patterson VC, Cumby J, et al. Affective lability in offspring of parents with major depressive disorder, bipolar disorder and schizophrenia. Eur Child Adolesc Psychiatr. (2020) 29:445-51. doi: 10.1007/s00787-019-01355-z

14. Oliver D, Spada G, Englund A, Chesney E, Radua J, Reichenberg A, et al. Real-world digital implementation of the psychosis polyrisk score (PPS): a pilot feasibility study. Schizophr Res. (2020) 226:176-83. doi: 10.1016/j.schres.2020.04.015

15. Docherty AR, Shabalin AA, Adkins DE, Mann F, Krueger RF, BacanuA, et al. Molecular genetic risk for psychosis is associated with psychosis risk symptoms in a population-based UK cohort: findings from generation scotland. Schizophr Bull. (2020) 46:1045-52. doi: 10.1093/schbul/sbaa042

16. Musliner KL, Krebs MD, Albiñana C, Vilhjalmsson B, Agerbo E, Zandi PP, et al. Polygenic risk progression to bipolar or psychotic disorders among individuals diagnosed with unipolar depression in early life. Am J Psychiatr. (2020) 177:936-43. doi: 10.1176/appi.ajp.2020.19111195

17. Watkeys OJ, Cohen-Woods S, Quidé Y, Cairns MJ, Overs B, Fullerton JM, et al. Derivation of poly-methylomic profile scores for schizophrenia. Prog Neuro Psychopharmacol Biol Psychiatr. (2020) 101:109925. doi: 10.1016/j.pnpbp.2020.109925

18. Bhak Y, Jeong H-O, Cho YS, Jeon S, Cho J, Gim JA, et al. Depression and suicide risk prediction models using blood-derived multi-omics data. Transl Psychiatr. (2019) 9:262. doi: 10.1038/s41398-019-0595-2

19. Bevins CL, Salzman NH. The potter's wheel: the host's role in sculpting its microbiota. Cell Mol Life Sci. (2011) 68:3675. doi: 10.1007/s00018-011-0830-3

20. Almeida A, Mitchell AL, Boland M, Forster SC, Gloor GB, Tarkowska A, et al. A new genomic blueprint of the human gut 
microbiota. Nature. (2019) 568:499-504. doi: 10.1038/s41586-019-0 965-1

21. Proctor LM, Creasy HH, Fettweis JM, Lloyd-Price J, Mahurkar A, Zhou W, et al. The integrative human microbiome project. Nature. (2019) 569:641-8. doi: 10.1038/s41586-019-1238-8

22. Scarpellini E, Campanale M, Leone D, Purchiaroni F, Vitale G, Lauritano EC, et al. Gut microbiota and obesity. Intern Emerg Med. (2010) 5:53-6. doi: 10.1007/s11739-010-0450-1

23. Mazmanian SK, Liu CH, Tzianabos AO, Kasper DL. An immunomodulatory molecule of symbiotic bacteria directs maturation of the host immune system. Cell. (2005) 122:107-18. doi: 10.1016/j.cell.2005.05.007

24. Spanogiannopoulos P, Bess EN, Carmody RN, Turnbaugh PJ. The microbial pharmacists within us: a metagenomic view of xenobiotic metabolism. Nat Rev Microbiol. (2016) 14:273. doi: 10.1038/nrmicro.2016.17

25. Johnson KV-A, Foster KR. Why does the microbiome affect behaviour? Nat Rev Microbiol. (2018) 16:647-55. doi: 10.1038/s41579-018-0014-3

26. Cuomo A, Maina G, Rosso G, Crescenzi BB, Bolognesi S, Muro A, et al. The microbiome: A new target for research and treatment of schizophrenia and its resistant presentations? A systematic literature search and review. Front Pharmacol. (2018) 9:1040. doi: 10.3389/fphar.2018.01040

27. Rodrigues-Amorim D, Rivera-Baltanás T, Regueiro B, Spuch C, de las Heras ME, Vázquez-Noguerol Méndez R, et al. The role of the gut microbiota in schizophrenia: current and future perspectives. World J Biol Psychiatr. (2018) 19:571-85. doi: 10.1080/15622975.2018.1433878

28. Painold A, Mörkl S, Kashofer K, Halwachs B, Dalkner N, Bengesser S, et al. A step ahead: exploring the gut microbiota in inpatients with bipolar disorder during a depressive episode. Bipolar Disord. (2019) 21:40-9. doi: $10.1111 /$ bdi.12682

29. Slyepchenko A, Maes M, Jacka FN, Köhler CA, Barichello T, McIntyre RS, et al. Gut microbiota, bacterial translocation, and interactions with diet: pathophysiological links between major depressive disorder and noncommunicable medical comorbidities. Psychother Psychosom. (2017) 86:3146. doi: $10.1159 / 000448957$

30. Cheung SG, Goldenthal AR, Uhlemann AC, Mann JJ, Miller JM, Sublette ME. Systematic review of gut microbiota and major depression. Front Psychiatr. (2019) 10:34. doi: 10.3389/fpsyt.2019.00034

31. Zhu F, Ju Y, Wang W, Wang Q, Guo R, Ma Q, et al. Metagenome-wide association of gut microbiome features for schizophrenia. Nat Commun. (2020) 11:1-10. doi: 10.1038/s41467-020-15457-9

32. Valles-Colomer M, Falony G, Darzi Y, Tigchelaar EF, Wang J, Tito RY, et al. The neuroactive potential of the human gut microbiota in quality of life and depression. Nat Microbiol. (2019) 4:623-32. doi: 10.1038/s41564-018-0337-x

33. Dinan TG, Borre YE, Cryan JF. Genomics of schizophrenia: time to consider the gut microbiome? Mol Psychiatr. (2014) 19:1252-7. doi: $10.1038 / \mathrm{mp} .2014 .93$

34. Borre YE, O'Keeffe GW, Clarke G, Stanton C, Dinan TG, Cryan JF. Microbiota and neurodevelopmental windows: implications for brain disorders. Trends Mol Med. (2014) 20:509-18. doi: 10.1016/j.molmed.2014.05.002

35. Heijtz RD, Wang S, Anuar F, Qian Y, Björkholm B, Samuelsson A, et al. Normal gut microbiota modulates brain development and behavior. Proc Natl Acad Sci USA. (2011) 108:3047-52. doi: 10.1073/pnas.1010529108

36. De Theije CGM, Wopereis H, Ramadan M, van Eijndthoven T, Lambert $\mathrm{J}$, Knol J, et al. Altered gut microbiota and activity in a murine model of autism spectrum disorders. Brain Behav Immun. (2014) 37:197-206. doi: 10.1016/j.bbi.2013.12.005

37. Buffington SA, Di Prisco GV, Auchtung TA, Ajami NJ, Petrosino JF, Costa-Mattioli M. Microbial reconstitution reverses maternal dietinduced social and synaptic deficits in offspring. Cell. (2016) 165:1762-75. doi: 10.1016/j.cell.2016.06.001

38. Degroote S, Hunting DJ, Baccarelli AA, Takser L. Maternal gut and fetal brain connection: increased anxiety and reduced social interactions in Wistar rat offspring following peri-conceptional antibiotic exposure. Prog Neuro Psychopharmacol Biol Psychiatr. (2016) 71:76-82. doi: 10.1016/j.pnpbp.2016.06.010

39. Tochitani $S$, Ikeno $T$, Ito $T$, Sakurai A, Yamauchi $T$, Matsuzaki $H$. Administration of non-absorbable antibiotics to pregnant mice to perturb the maternal gut microbiota is associated with alterations in offspring behavior. PLoS ONE. (2016) 11:e0138293. doi: 10.1371/journal.pone.0138293
40. Golubeva AV, Crampton S, Desbonnet L, Edge D, O'Sullivan $\mathrm{O}$, Lomasney $\mathrm{KW}$, et al. Prenatal stress-induced alterations in major physiological systems correlate with gut microbiota composition in adulthood. Psychoneuroendocrinology. (2015) 60:58-74. doi: 10.1016/j.psyneuen.2015.06.002

41. Gur TL, Shay L, Palkar AV, Fisher S, Varaljay VA, Dowd S, et al. Prenatal stress affects placental cytokines and neurotrophins, commensal microbes, and anxiety-like behavior in adult female offspring. Brain Behav Immun. (2017) 64:50-8. doi: 10.1016/j.bbi.2016.12.021

42. Jašarević E, Howard CD, Misic AM, Beiting DP, Bale TL. Stress during pregnancy alters temporal and spatial dynamics of the maternal and offspring microbiome in a sex-specific manner. Sci Rep. (2017) 7:44182. doi: $10.1038 /$ srep 44182

43. Lozupone CA, Stombaugh JI, Gordon JI, Jansson JK, Knight R. Diversity, stability and resilience of the human gut microbiota. Nature. (2012) 489:22030. doi: 10.1038/nature11550

44. Maynard CL, Elson CO, Hatton RD, Weaver CT. Reciprocal interactions of the intestinal microbiota and immune system. Nature. (2012) 489:231-41. doi: 10.1038/nature11551

45. Parfrey LW, Knight R. Spatial and temporal variability of the human microbiota. Clin Microbiol Infect. (2012) 18:5-7. doi: 10.1111/j.1469-0691.2012.03861.x

46. Sudo N, Chida Y, Aiba Y, Sonoda J, Oyama N, Yu X, et al. Postnatal microbial colonization programs the hypothalamic-pituitary-adrenal system for stress response in mice. J Physiol. (2004) 558:263-75. doi: 10.1113/jphysiol.2004.063388

47. Clarke G, Grenham S, Scully P, Fitzgerald P, Moloney RD, Shanahan F, et al. The microbiome-gut-brain axis during early life regulates the hippocampal serotonergic system in a sex-dependent manner. Mol Psychiatr. (2013) 18:66673. doi: $10.1038 / \mathrm{mp} .2012 .77$

48. Hoban AE, Stilling RM, Ryan FJ, Shanahan F, Dinan TG, Claesson MJ, et al. Regulation of prefrontal cortex myelination by the microbiota. Transl Psychiatr. (2016) 6:e774. doi: 10.1038/tp.2016.42

49. Desbonnet L, Clarke G, Shanahan F, Dinan TG, Cryan JF. Microbiota is essential for social development in the mouse. Mol Psychiatr. (2014) 19:146-8. doi: $10.1038 / \mathrm{mp} .2013 .65$

50. Erny D, de Angelis ALH, Jaitin D, Wieghofer P, Staszewski O, David E, et al. Host microbiota constantly control maturation and function of microglia in the CNS. Nat Neurosci. (2015) 18:965-77. doi: 10.1038/nn.4030

51. He Y, Kosciolek T, Tang J, Zhou Y, Li Z, Ma X, et al. Gut microbiome and magnetic resonance spectroscopy study of subjects at ultra-high risk for psychosis may support the membrane hypothesis. Eur Psychiatr. (2018) 53:37-45. doi: 10.1016/j.eurpsy.2018.05.011

52. de Angelis M, Piccolo M, Vannini L, Siragusa S, De Giacomo A, Serrazzanetti DI, et al. Fecal microbiota and metabolome of children with autism and pervasive developmental disorder not otherwise specified. PLoS ONE. (2013) 8:e76993. doi: 10.1371/journal.pone.0076993

53. Shen Y, Xu J, Li Z, Huang Y, Yuan Y, Wang J, et al. Analysis of gut microbiota diversity and auxiliary diagnosis as a biomarker in patients with schizophrenia: a cross-sectional study. Schizophr Res. (2018) 197:470-7. doi: 10.1016/j.schres.2018.01.002

54. Koh A, De Vadder F, Kovatcheva-Datchary P, Bäckhed F. From dietary fiber to host physiology: short-chain fatty acids as key bacterial metabolites. Cell. (2016) 165:1332-45. doi: 10.1016/j.cell.2016.05.041

55. Braniste V, Al-Asmakh M, Kowal C, Anuar F, Abbaspour A, Tóth M, et al. The gut microbiota influences blood-brain barrier permeability in mice. Sci Transl Med. (2014) 6:263ra158. doi: 10.1126/scitranslmed.3009759

56. Frost G, Sleeth ML, Sahuri-Arisoylu M, Lizarbe B, Cerdan S, Brody L, et al. The short-chain fatty acid acetate reduces appetite via a central homeostatic mechanism. Nat Commun. (2014) 5:4611. doi: 10.1038/ncomms4611

57. Anderson G, Maes M. Gut dysbiosis dysregulates central and systemic homeostasis via suboptimal mitochondrial function: assessment, treatment and classification implications. Curr Top Med Chem. (2020) 20:524-39. doi: 10.2174/1568026620666200131094445

58. Lowry CA, Smith DG, Siebler PH, Schmidt D, Stamper CE, Hassell JE, et al. The microbiota, immunoregulation, and mental health: implications for public health. Curr Environ Heal Rep. (2016) 3:270-86. doi: $10.1007 / \mathrm{s} 40572-016-0100-5$ 
59. Warner BB. The contribution of the gut microbiome to neurodevelopment and neuropsychiatric disorders. Pediatr Res. (2019) 85:216-24. doi: 10.1038/s41390-018-0191-9

60. Rook GAW, Raison CL, Lowry CA. Microbial 'old friends', immunoregulation and socioeconomic status. Clin Exp Immunol. (2014) 177:1-12. doi: $10.1111 /$ cei. 12269

61. Choi GB, Yim YS, Wong H, Kim S, Kim H, Kim SV, et al. The maternal interleukin-17a pathway in mice promotes autism-like phenotypes in offspring. Science. (2016) 351:933-9. doi: 10.1126/science.aad0314

62. Houser MC, Tansey MG. The gut-brain axis: Is intestinal inflammation a silent driver of Parkinson's disease pathogenesis? NPJ Park Dis. (2017) 3:3. doi: 10.1038/s41531-016-0002-0

63. Sampson TR, Debelius JW, Thron T, Janssen S, Shastri GG, Ilhan ZE, et al. Gut microbiota regulate motor deficits and neuroinflammation in a model of Parkinson's disease. Cell. (2016) 167:1469-80. doi: 10.1016/j.cell.2016.11.018

64. Lu B, Nagappan G, Lu Y. BDNF synaptic plasticity. cognitive function, and dysfunction. Handb Exp Pharmacol. (2015) 220:223-50. doi: 10.1007/978-3-642-45106-5_9

65. Atladóttir HÓ, Thorsen P, Østergaard L, Schendel DE, Lemcke S, Abdallah $\mathrm{M}$, et al. Maternal infection requiring hospitalization during pregnancy autism spectrum disorders. J Autism Dev Disord. (2010) 40:1423-30. doi: 10.1007/s10803-010-1006-y

66. Atladóttir HÓ, Henriksen TB, Schendel DE, Parner ET. Autism after infection, febrile episodes, and antibiotic use during pregnancy: an exploratory study. Pediatrics. (2012) 130:e1447-54. doi: 10.1542/peds.2012-1107

67. Li M, Chang H, Xiao X. BDNF Val66Met polymorphism and bipolar disorder in European populations: a risk association in case-control, family-based and GWAS studies. Neurosci Biobehav Rev. (2016) 68:218-33. doi: 10.1016/j.neubiorev.2016.05.031

68. Di Carlo P, Punzi G, Ursini G. Brain-derived neurotrophic factor and schizophrenia. Psychiatr Genet. (2020) 29:200-10. doi: 10.1097/YPG.0000000000000237

69. Karim HT, Wang M, Andreescu C, Tudorascu D, Butters MA, Karp JF, et al. Acute trajectories of neural activation predict remission to pharmacotherapy in late-life depression. NeuroImage Clin. (2018) 19:831-9. doi: 10.1016/j.nicl.2018.06.006

70. Zhou Y, Fan L, Qiu C, Jiang T. Prefrontal cortex and the dysconnectivity hypothesis of schizophrenia. Neurosci Bull. (2015) 31:207-19. doi: 10.1007/s12264-014-1502-8

71. Janiri D, Moser DA, Doucet GE, Luber MJ, Rasgon A, Lee WH, et al. Shared neural phenotypes for mood and anxiety disorders: a meta-analysis of 226 task-related functional imaging studies. JAMA Psychiatr. (2020) 77:172-9. doi: 10.1001/jamapsychiatry.2019.3351

72. Phillips ML, Swartz HA. A critical appraisal of neuroimaging studies of bipolar disorder: toward a new conceptualization of underlying neural circuitry and a road map for future research. Am J Psychiatr. (2014) 171:829-43. doi: 10.1176/appi.ajp.2014.13081008
73. Rapinesi C, Del Casale A, Di Pietro S, Ferri VR, Piacentino D, Sani G, et al Add-on high frequency deep transcranial magnetic stimulation (dTMS) to bilateral prefrontal cortex reduces cocaine craving in patients with cocaine use disorder. Neurosci Lett. (2016) 629:43-7. doi: 10.1016/j.neulet.2016.06.049

74. Takahashi N, Sakurai T, Davis KL, Buxbaum JD. Linking oligodendrocyte and myelin dysfunction to neurocircuitry abnormalities in schizophrenia. Prog Neurobiol. (2011) 93:13-24. doi: 10.1016/j.pneurobio.2010.09.004

75. Wodarz A, Nusse R. Mechanisms of Wnt signaling in development. Annu Rev Cell Dev Biol. (1998) 14:59-88. doi: 10.1146/annurev.cellbio.14.1.59

76. Cotter D, Kerwin R, Al-Sarraji S, Brion JP, Chadwich A, Lovestone $\mathrm{S}$, et al. Abnormalities of Wnt signalling in schizophrenia-evidence for neurodevelopmental abnormality. Neuroreport. (1998) 9:1379-83. doi: 10.1097/00001756-199805110-00024

77. Hur EM, Zhou FQ. GSK3 signalling in neural development. Nat Rev Neurosci. (2010) 11:539-51. doi: 10.1038/nrn2870

78. Sani G, Napoletano F, Maria Forte A, Kotzalidis G, Panaccione I, Maria Porfiri G, et al. The wnt pathway in mood disorders. Curr Neuropharmacol. (2012) 10:239-53. doi: 10.2174/157015912803217279

79. Panaccione I, Napoletano F, Maria Forte A, Kotzalidis G, Del Casale A, Rapinesi C, et al. Neurodevelopment in schizophrenia: the role of the wnt pathways. Curr Neuropharmacol. (2013) 11:535-58. doi: 10.2174/1570159X113119990037

80. Moossavi S. Location-specific effect of microbiota and MyD88-dependent signaling on Wnt/ $\beta$-catenin pathway and intestinal stem cells. Gut Microbes. (2014) 5:11-4. doi: 10.4161/gmic.27291

81. Simeonova D, Ivanovska $M$, Murdjeva $M$, Carvalho AF, Maes $M$. Recognizing the leaky gut as a trans-diagnostic target for neuro-immune disorders using clinical chemistry and molecular immunology assays. Curr Top Med Chem. (2018) 18:1641-55. doi: 10.2174/15680266186661811151 00610

82. Manchia M, Squassina A, Pisanu C, Congiu D, Garzilli M, Guiso B, et al. Investigating the relationship between melatonin levels, melatonin system, microbiota composition and bipolar disorder psychopathology across the different phases of the disease. Int J Bipolar Disord. (2019) 7:27. doi: 10.1186/s40345-019-0163-y

Conflict of Interest: The authors declare that the research was conducted in the absence of any commercial or financial relationships that could be construed as a potential conflict of interest.

Copyright () 2021 Sani, Manchia, Simonetti, Janiri, Paribello, Pinna and Carpiniello. This is an open-access article distributed under the terms of the Creative Commons Attribution License (CC BY). The use, distribution or reproduction in other forums is permitted, provided the original author(s) and the copyright owner(s) are credited and that the original publication in this journal is cited, in accordance with accepted academic practice. No use, distribution or reproduction is permitted which does not comply with these terms. 